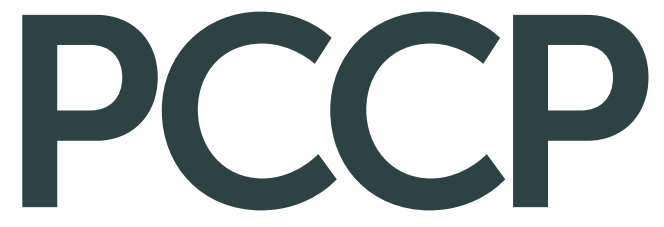

Physical Chemistry Chemical Physics rsc.li/pccp
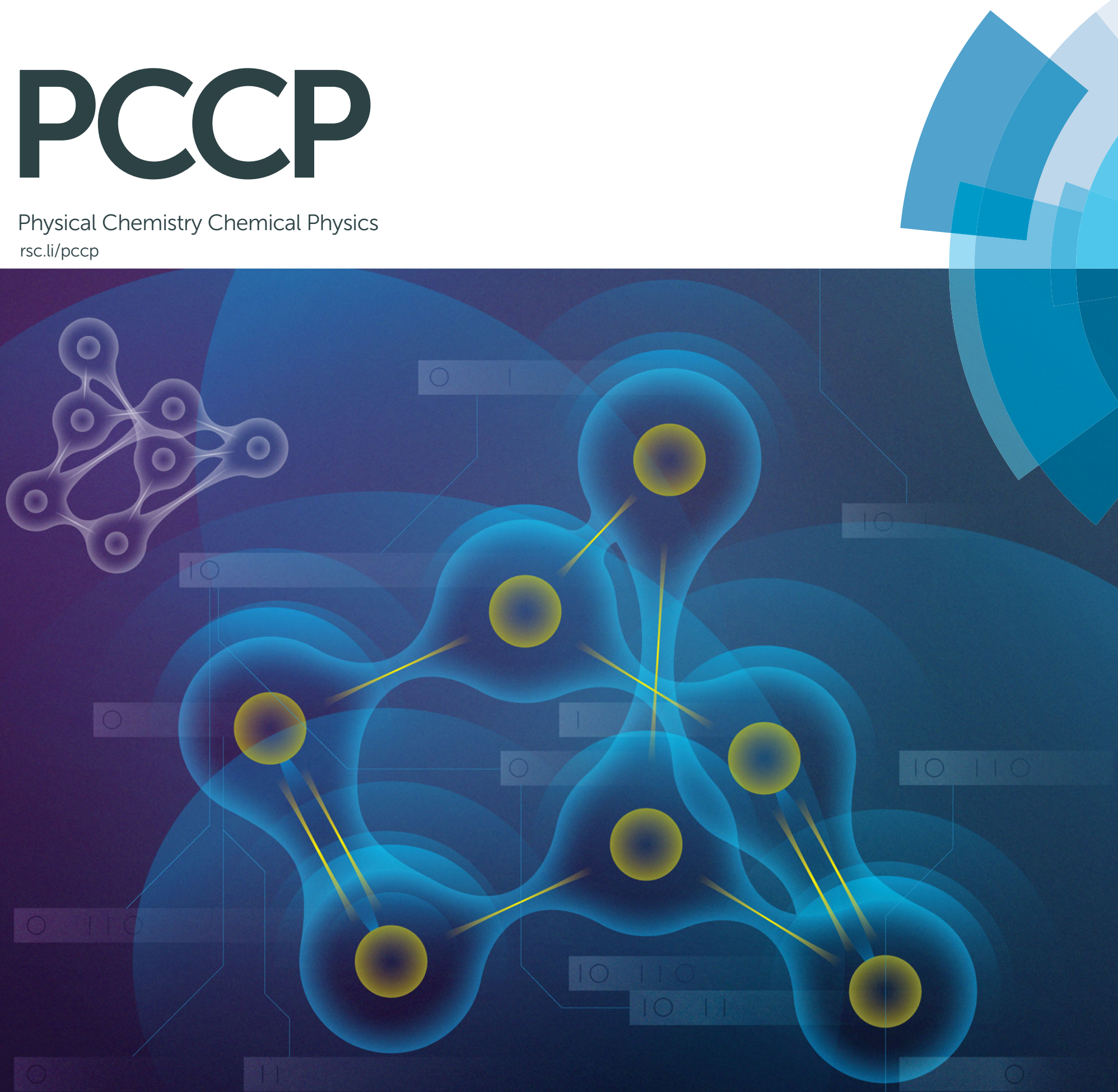

ISSN 1463-9076 
Check for updates

Cite this: Phys. Chem. Chem. Phys. 2018, 20, 23195

Received 9th July 2018 Accepted 15th August 2018

DOI: $10.1039 / c 8 c p 04329 a$

rsc.li/pccp

\section{Turn-off mode fluorescent norbornadiene-based photoswitches $\dagger$}

\author{
Behabitu Ergette Tebikachew, (D) Fredrik Edhborg, (D) Nina Kann, (D) \\ Bo Albinsson (D) * and Kasper Moth-Poulsen (D) *
}

\section{Introduction}

The emission of light from certain photoresponsive materials upon photoexcitation has intrigued scientists in the past and led to a variety of applications. ${ }^{1,2}$ More recently, however, the ability to modulate the emission of the emitting species has drawn significant attention, as it lends itself for applications ranging from biological imaging to ultra-high density optical memories. ${ }^{4}$ In this context, photochromic molecules present an attractive opportunity since they can be tailored towards a specific application.

Photochromic molecules are molecules that change their colour reversibly upon irradiation with light. This colour change arises from a change in the structural and stereoelectronic arrangement in the molecule. Besides the colour change, a change in absorption, ${ }^{5}$ electrical conductance ${ }^{4,6}$ and dipole moment $^{7,8}$ of the photochromic molecule can be manifested.

Department of Chemistry and Chemical Engineering, Chalmers University of

Technology, SE-41296 Gothenburg, Sweden. E-mail: balb@chalmers.se,

mkasper@chalmers.se

$\dagger$ Electronic supplementary information (ESI) available: Synthetic procedures,

${ }^{1} \mathrm{H},{ }^{13} \mathrm{C}$ and COSY-NMR spectra, UV-Vis absorption, kinetics and Arrhenius analysis. Photoisomerization quantum yield, fluorescence lifetime measurements and TDDFT calculations data. See DOI: 10.1039/c8cp04329a
Some photochromic molecules are intrinsically fluorescent in one form and non-fluorescent in the other isomer. Another way to impart fluorescence properties to a photochromic molecule is by attaching a fluorophore. ${ }^{9-11}$ In the latter case, the absorption of one of the isomers of the photochromic unit needs to be matched to the emission of the fluorophore so that efficient quenching occurs. Upon light-triggered photoisomerization, the fluorescence can be switched back on. This light induced modulation of the emissive properties of a photochromic molecule between the fluorescent and the non-fluorescent form has been successfully used in high-resolution fluorescence imaging techniques $^{12-15}$ as well as ultrahigh density optical memories. ${ }^{16-18}$ For a fluorescent photochromic molecule to be used for such types of applications, certain requirements need to be met. Two of the most critical ones are fatigue resistance and high fluorescence quantum yield. ${ }^{4,5}$

Various fluorescent photochromic molecules have been reported in the literature, ${ }^{4,7,19-23}$ where the most prominent so far has been the diarylethene (DAE)-based systems. Since most DAEs are not intrinsically fluorescent, a fluorescent unit such as anthracene, ${ }^{24}$ organic dyes ${ }^{25}$ or even nanoparticles ${ }^{9}$ have been attached to the DAE photochromic unit. Some DAE derivatives, e.g. benzothiophenes, are fluorescent in themselves; however, they suffer from poor fluorescence quantum yields. Recently, certain highly fluorescent 


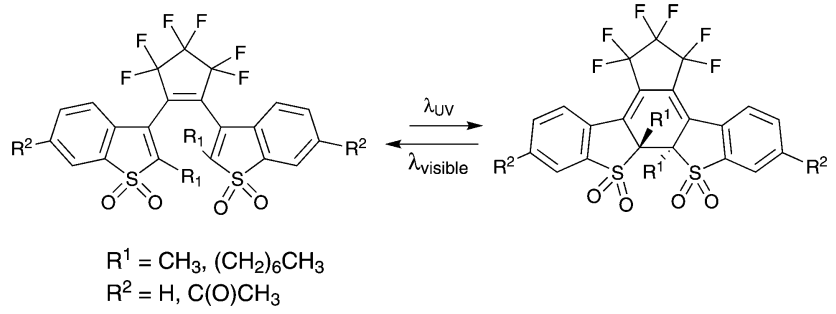

Scheme 1 Examples of fluorescent DAE-based molecules. ${ }^{22}$

DAE derivatives with a fluorescent quantum yield close to $90 \%$ were prepared by oxidizing the sulphur on the thiophene unit of the DAE to a sulphur-S,S-dioxide (Scheme 1) ${ }^{26}$ However, the photostability of these dioxides is reported to be lower than the unoxidized counterparts. ${ }^{23}$

Another photochromic molecule that has seen a renewed interest recently due to possible applications in solar energy storage $^{27-30}$ and data storage ${ }^{3}$ is the norbornadiene-quadricyclane (NBD-QC) system (Scheme 2). The NBD-QC pair is a T-type ${ }^{5}$ negative photochrome, where the unsaturated diene, i.e. the NBD-form, converts to the saturated QC-form upon photoirradiation. When desired, the QC-form can be triggered to relax back to the NBD-form thermally, ${ }^{27}$ electrochemically, ${ }^{30}$ by catalytic activation ${ }^{31}$ or even using light. ${ }^{32-34}$ This class of photochromic molecules has seen a recent rise in interest, since a relatively low energy photon is used to initiate the photoisomerization of the parent molecule to its isomer compared to positive photochromes. ${ }^{35}$ This is particularly attractive for biological applications as it is less damaging to cells and tissues.

There are few reported examples of fluorescent NBD-QC photochromic systems. Maafi et al. described one of the earliest examples of the fluorescence properties of norbornadiene derivatives containing an ester unit (Scheme 2) ${ }^{36}$ Interestingly, upon photoisomerization to its QC-form, the emission intensity increased. The lower emission in the NBD-form was attributed to intramolecular self-quenching of the phenyl ester fluorophore emission by the NBD unit. A similar behaviour was observed in a polyphenylene polymer containing a pendant norbornadiene moiety. ${ }^{37}$ Babudri et al. also reported a conjugated polymer containing an NBD backbone structure with a fluorescent quantum yield of $24 \% .^{38}$ However, long exposure to UV irradiation resulted in a significant decrease in the fluorescence quantum yield.

In the synthesis of various 2,3-disubstituted norbornadienequadricyclane (NBD-QC) photochromic systems, we have observed fluorescence in certain NBD-derivatives. ${ }^{3}$ Here, we report unprecedented emission properties for this class of photochromic systems,

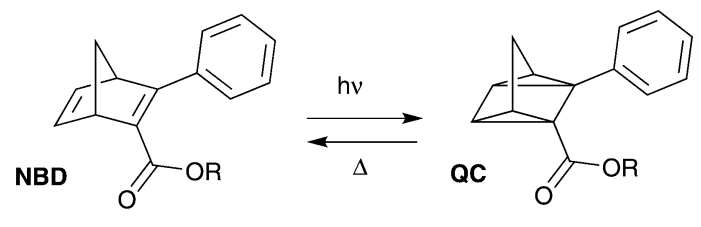

$$
\mathrm{R}=\mathrm{H}, \mathrm{CH}_{2} \mathrm{Ph}, \mathrm{CH}_{2} \mathrm{C}_{6} \mathrm{H}_{4} \mathrm{OMe}-4,\left(\mathrm{CH}_{2}\right)_{6} \mathrm{C}_{6} \mathrm{H}_{4} \mathrm{OMe}
$$

Scheme 2 Examples of fluorescent NBD-QC systems. ${ }^{37}$ featuring a high fluorescence quantum yield in the NBD-form, a virtually non-emissive QC-form and excellent fatigue resistance. Furthermore, we demonstrate fluorescence modulation through multiple emission switching cycles by photoisomerization from NBD to QC and thermally activated back-conversion. Moreover, we also show the NBD to QC and QC to NBD switching, using relatively low energy UV (340 $\mathrm{nm})$ sources, making this an entirely photoswitching system.

\section{Experimental details}

\section{General procedures}

All reagents and HPLC grade solvents were obtained from commercial sources and used as received. Dry solvents (toluene and tetrahydrofuran) were obtained from MBraun MB SPS-800 solvent purification system. All reactions were performed in oven-dried flasks under positive nitrogen pressure unless stated otherwise. Flash chromatography was conducted using a Biotage Isolera $^{\mathrm{TM}}$ Spektra One flash chromatography system. Thin-layer chromatography (TLC) was performed on pre-coated aluminum plates with Merck Silica gel $60 \mathrm{~F}_{254}$ and the spots were visualized by using 254 and $365 \mathrm{~nm}$ handheld UV lamp. Infrared measurements were carried out using Perkin Elmer Frontier instruments provided with an ATR module.

Proton $\left({ }^{1} \mathrm{H}\right)$, carbon $\left({ }^{13} \mathrm{C}\right)$ and correlation spectroscopy (COSY) nuclear magnetic resonance (NMR) were recorded on an automated Agilent (Varian) MR 400 (400 MHz) spectrometer equipped with a "One-probe". Chemical shifts are given in parts per million ( $\mathrm{ppm}$ ) downfield from tetramethylsilane referring to the residual proton signal $\left(\mathrm{CHCl}_{3}: 7.26 \mathrm{ppm}\right.$ in $\left.{ }^{1} \mathrm{H}-\mathrm{NMR}\right)$ and carbon signal $\left(\mathrm{CDCl}_{3}: 77.0 \mathrm{ppm}\right.$ in $\left.{ }^{13} \mathrm{C}-\mathrm{NMR}\right)$ in the NMR solvent. Data are presented as chemical shift, multiplicity ( $\mathrm{s}=$ singlet, $\mathrm{d}=$ doublet, $\mathrm{t}=$ triplet, $\mathrm{q}=$ quartet, $\mathrm{m}=$ multiplet), coupling constants in Hertz $(\mathrm{Hz})$ and integration. Absorption spectroscopy and kinetics experiments were carried out using a Cary 50, Cary 100 or Cary 4000 UV-vis spectrophotometer provided with a water heating system. Fluorescence emission spectra were recorded on a Spex Fluorolog 3 spectrofluorimeter from JY Horiba. Photoswitching experiments were performed using Thorlabs LED lamps $340 \mathrm{~nm}$ (M340L4), $365 \mathrm{~nm}$ (M365F1) and $405 \mathrm{~nm}$ (M405LP1). Photoisomerization quantum yields were measured according to a published literature method $^{39}$ using a concentrated sample (absorbance $>2$ ) at room temperature. The details are provided in Section V of the ESI. $\dagger$ High resolution mass (HRMS) was obtained using Agilent 1290 Infinity LC system tandem to an Agilent 6520 Accurate Mass Q-TOF LC/MS with an APCI source in a positive mode. Elemental analyses were performed at Mikrolab Kolbe.

The fluorescence quantum yield $\left(\Phi_{\mathrm{F}}\right)$ was measured using the procedure described in literature ${ }^{40}$ with Coumarin 102 in ethanol $\left(\Phi_{\mathrm{F}}=0.80\right)$ as reference. The concentration of the sample and reference sample was chosen so that both had the same absorption at the excitation wavelength. Excited state lifetime measurements were carried out using time correlated single photo counting (TCSPC) with a $377 \mathrm{~nm}$ excitation source 
from PicoQuant. An MCP-PMT detector was used, with 2048 channels and 10000 counts in the top channel. The decay curves were fitted to mono- or bi-exponentials decays by deconvolution with the instrument response function. Vertical excitation energies and oscillator strengths were calculated by employing time dependent DFT (TDDFT) on DFT//B3LYP/6$31 G^{* *}$ optimized structures.

\section{General procedure for the synthesis of the NBD compounds}

In an oven-dried Schlenk flask (25 $\mathrm{mL})$ provided with magnetic stirring bar, were added $\mathrm{Pd}\left(\mathrm{PPh}_{3}\right)_{4}$ (5 mol\%), CuI (10 mol\%), 2,3-dibromonorbornadiene (1.2 mmol, 1 eq.) and toluene $(10 \mathrm{~mL})$. The solution was purged with nitrogen for 15 minutes. Then, diisopropylamine $(1 \mathrm{~mL})$ and the alkyne $(2.6 \mathrm{mmol}$, 2.1 eq.) were added, at which point the colour of the solution turned from yellow to dark red. The mixture was reacted until the starting materials were consumed as indicated by TLC. The solution was then diluted with dichloromethane and filtered through a short silica gel plug. The volatiles were then removed using a rotary evaporator. The crude product obtained was submitted to automated flash chromatography using an eluent gradient of $0-5 \%$ dichloromethane in hexane. The product was obtained after the solvent was removed in vacuo. (For the detailed synthetic procedure and characterization, see the ESI $\dagger$ ).

\section{Results and discussion}

\section{Synthesis}

The synthesis of the NBD-derivatives, symmetrically substituted with a thiophenyl moiety as well as a thioacetate-terminated bis(ethynylphenylene), are shown in Scheme 3. The synthesis employed 2,3-dibromonorbornadiene (11) as the starting material, which in turn, was synthesized from bicyclo[2.2.1]hepta-2,5diene (2,5-norbornadiene) according to a literature procedure. ${ }^{3}$ Compounds 1 and 2 were obtained in good yields (ca. 60\%) via a Sonogashira reaction of 2- or 3-ethynylthiophene with 11 .
The synthesis of $\mathbf{3}$ and $\mathbf{4}$ was achieved by first preparing $\mathbf{5}$ and 6 from the corresponding commercially available bromoiodobenzenes via a double Sonogashira reaction. Deprotection of the trimethylsilyl groups furnished the dialkynylated compounds 7 and 8 in excellent yields $(>90 \%)$. Subsequent Sonogashira reaction of 7 and $\mathbf{8}$ with 11 led to the triisopropyl silyl (TIPS) protected intermediates $\mathbf{9}$ and 10. Ultimately, deprotection of the TIPS groups using tributylammonium fluoride (TBAF), followed by a Sonogashira coupling with 4-iodophenyl thioacetate, yielded 3 and 4 in approximately 20 and $40 \%$ yield, respectively. It is worth noting that the synthesis of $\mathbf{4}$ has been described previously. ${ }^{3}$ In addition, all four compounds were characterized by elemental analysis, HRMS, IR and NMR (see ESI $\dagger$ ).

\section{Steady state absorption spectra}

The UV-vis absorption spectra of 1-4 in the NBD-form and QC-form were measured in toluene, see Fig. 1. For compounds 1-NBD and 4-NBD, that have the longest linearly conjugated systems, redshifted absorption spectra were observed in comparison to the respective cross-conjugated systems 2-NBD and 3-NBD. There is approximately a $50 \mathrm{~nm}$ difference between the onset of absorption (defined as $\log (\varepsilon)=2$ ) of the most redshifted spectrum of 4-NBD compared to that of 2-NBD, see Table 1 . The absorption spectrum of 4-NBD is not only redshifted, but also has the highest molar absorptivity of the four compounds studied (see ESI, $\dagger$ Fig. S13). The molar absorption coefficient $(\varepsilon)\left(43000 \mathrm{M}^{-1} \mathrm{~cm}^{-1}\right)$ of 4-NBD at the first maximum $\left(\lambda_{\max 1}=391 \mathrm{~nm}\right)$ is about two times higher than the other three compounds and comparable to some of the strongest diarylethene absorbers reported in the literature. ${ }^{26}$

\section{Kinetics and photoisomerization quantum yields $\left(\Phi_{\mathrm{NBD}-\mathrm{QC}}\right)$}

Irradiating compounds 1-NBD and 4-NBD with $405 \mathrm{~nm}$ light, and 2-NBD and 3-NBD with $365 \mathrm{~nm}$ light, converts the molecules 1-4 to their respective QC-forms with near quantitative conversion, as shown in Fig. 1. Moreover, no photostationary state was observed in any of the compounds studied, due to the strong blueshift in

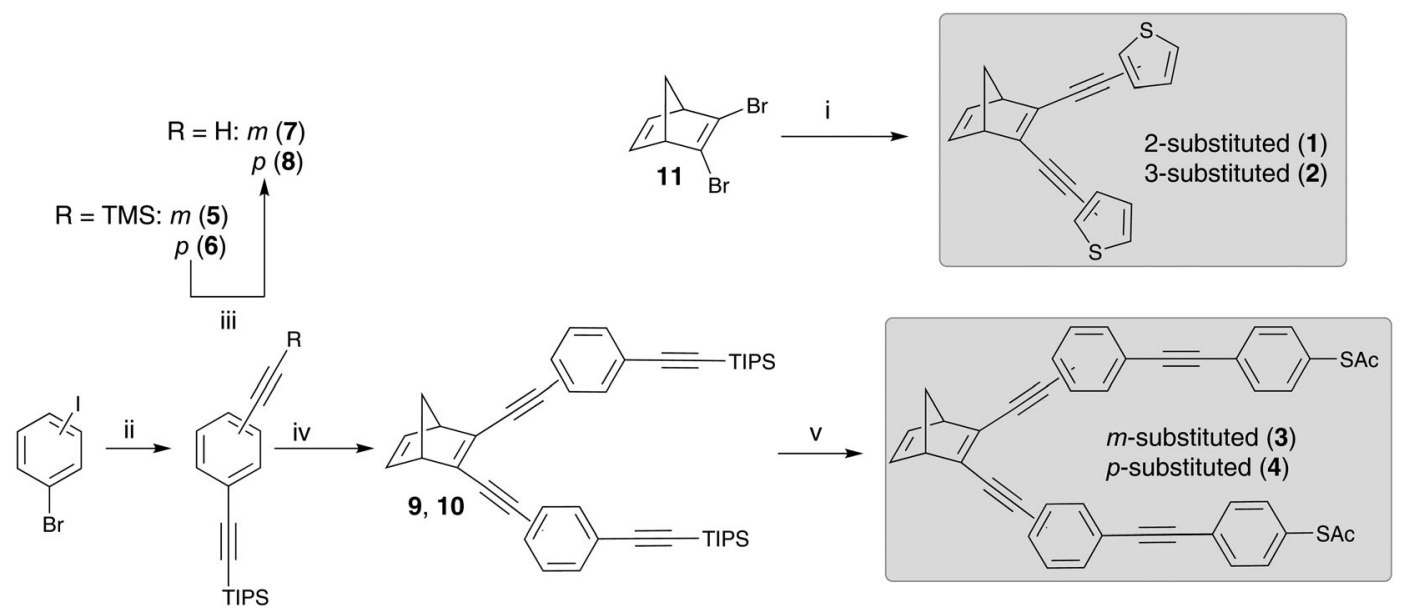

Scheme 3 Synthetic routes to the norbornadiene derivatives 1-4 starting from bicyclo[2.2.1]hepta-2,5-diene (11): (i) 2-ethynyl thiophene (for 1) or 3-ethynyl thiophene (for 2), $\mathrm{Pd}\left(\mathrm{PPh}_{3}\right) \mathrm{Cl}_{2}, \mathrm{Cul}, \mathrm{DIPA}, 35^{\circ} \mathrm{C}$; (ii) (a) triisopropylsilyl acetylene, $\mathrm{Pd}\left(\mathrm{PPh}_{3}\right)_{2} \mathrm{Cl}_{2}, \mathrm{Cul}$, DIPA, r.t., (b) trimethylsilyl acetylene, $60{ }^{\circ} \mathrm{C}$, $12 \mathrm{~h}$; (iii) $\mathrm{K}_{2} \mathrm{CO}_{3}, \mathrm{MeOH}: \mathrm{DCM}$, r.t.; (iv) 11, $\mathrm{Pd}\left(\mathrm{PPh}_{3}\right)_{2} \mathrm{Cl}_{2}, \mathrm{Cul}$, toluene, $\mathrm{Et}_{3} \mathrm{~N}, 35^{\circ} \mathrm{C}$; (v) (a) $\mathrm{TBAF}, 0{ }^{\circ} \mathrm{C}$ to r.t., (b) 4-(iodophenyl)thioacetate, $\mathrm{Pd}(\mathrm{PPh})_{4}$, $\mathrm{Cul}$, toluene, $50{ }^{\circ} \mathrm{C}$. 


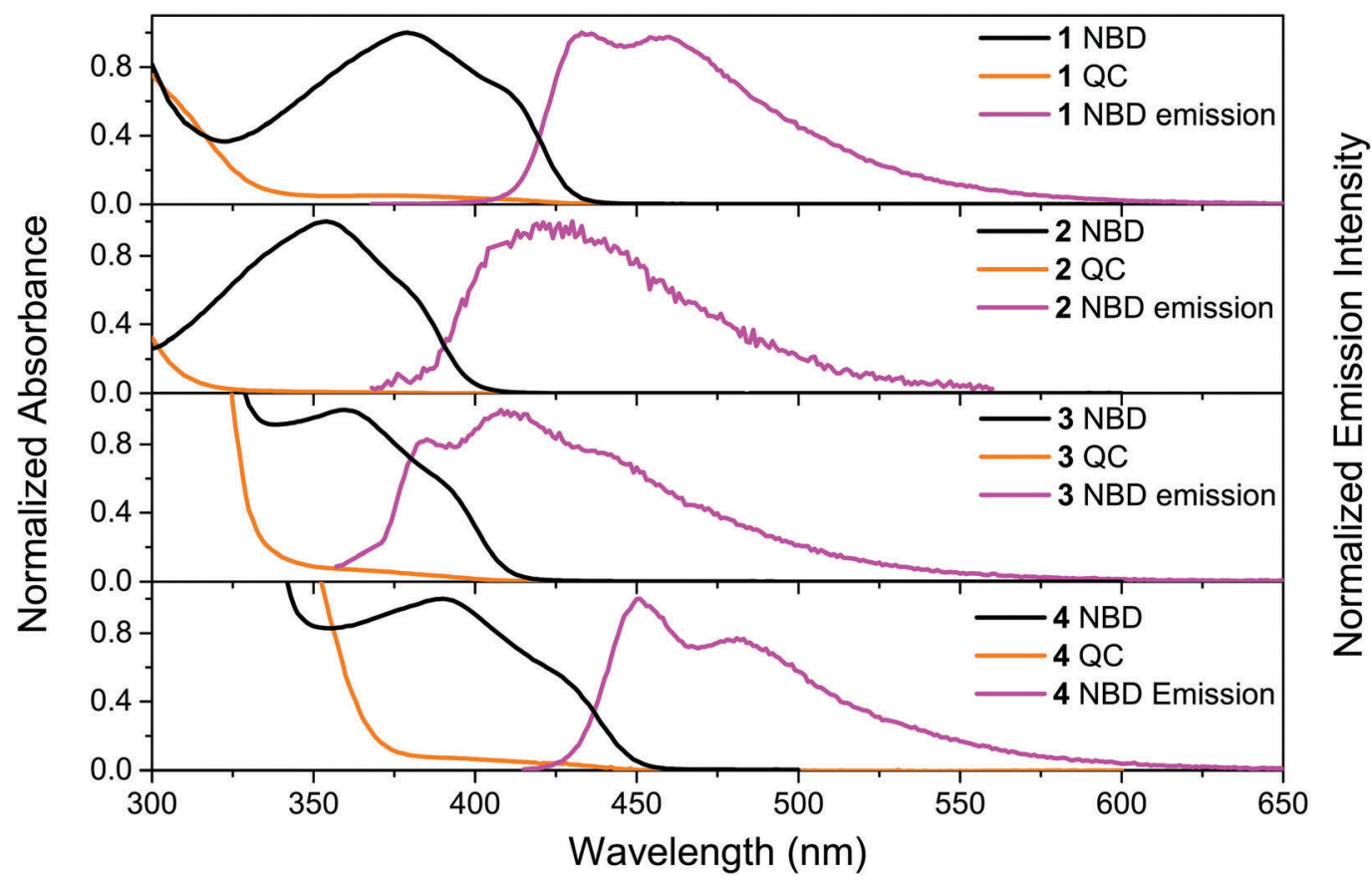

Fig. 1 Normalized steady state absorption spectra (NBD- and QC-forms) of 1, 2, 3 and $\mathbf{4}$ and the corresponding emission spectra ( $\lambda_{\text {ex }}=364,362,330$ and $407 \mathrm{~nm}$ ) in toluene.

Table 1 Summarized photophysical properties of $\mathbf{1 - 4}$ in toluene

\begin{tabular}{|c|c|c|c|c|c|c|c|c|}
\hline Compound & $\lambda_{\max 1}{ }^{a}(\mathrm{~nm})\left(\varepsilon\left(\mathrm{M}^{-1} \mathrm{~cm}^{-1}\right)\right) ; \lambda_{\text {onset }}^{b}(\mathrm{~nm})$ & $t_{1 / 2,25^{\circ} \mathrm{C}}{ }^{c}(\mathrm{~min})$ & $E_{\mathrm{a}}^{d}\left(\mathrm{~kJ} \mathrm{~mol}^{-1}\right)$ & $\Phi_{\mathrm{NBD} \rightarrow \mathrm{QC}}{ }^{e}$ & $\Phi_{\mathrm{F}}^{f}$ & $\tau_{\mathrm{F}}^{g}(\mathrm{~ns})$ & $k_{\mathrm{F}}^{h}\left(\mathrm{~s}^{-1}\right)$ & $k_{\mathrm{nr}}^{i}\left(\mathrm{~s}^{-1}\right)$ \\
\hline 2 & $357\left(1.8 \times 10^{4}\right) ; 415$ & 201 & 103.7 & 0.54 & 0.002 & 0.02 & $1.0 \times 10^{8}$ & $5.0 \times 10^{10}$ \\
\hline 3 & $\begin{array}{l}364\left(1.5 \times 10^{4}\right) \\
301\left(5.0 \times 10^{4}\right) ; 424\end{array}$ & 130 & 135.6 & 0.77 & 0.01 & 0.02 & $4.3 \times 10^{8}$ & $5.0 \times 10^{10}$ \\
\hline 4 & $\begin{array}{l}391\left(4.3 \times 10^{4}\right) \\
332\left(8.3 \times 10^{4}\right) ; 462\end{array}$ & 78.6 & 100.7 & 0.15 & 0.49 & 1.28 & $3.9 \times 10^{8}$ & $3.9 \times 10^{8}$ \\
\hline
\end{tabular}

${ }^{a}$ The first absorption maximum of NBD-forms. ${ }^{b}$ Absorption onset of the NBD-form is defined as the wavelength at which the molar absorptivity is $\log (\varepsilon) \approx 2 .{ }^{c}$ Half-life of the thermal back reaction QC $\rightarrow$ NBD-forms at $25{ }^{\circ} \mathrm{C} .{ }^{d}$ Activation energy for the thermal back reaction of QC $\rightarrow$ NBDforms. ${ }^{e}$ Quantum yield for the photoisomerization of NBD-form to QC-form. The values given are the average of two measurements (see ESI Section IV). ${ }^{f}$ Fluorescence quantum yield of NBD-forms. The values given are the average of three measurements (see ESI Section V). ${ }_{g}^{g}$ Fluorescence lifetime of NBD-forms. ${ }^{h}$ Radiative rate constant. ${ }^{i}$ Nonradiative rate constant.

absorption onset of the QC-form relative to the NBD-form. Since the NBD-QC pair is a thermal photoswitch system, the thermal recovery of the QC-forms of 1-4 was also measured (see ESI, $\dagger$ Section III). The corresponding Arrhenius parameters and halflives are presented in Table 1 . All four compounds have relatively short half-lives ( $c a .1 \mathrm{~h}$ to $3 \mathrm{~h}$ at $25{ }^{\circ} \mathrm{C}$ ) as a result of the low average activation energy $\left(E_{\mathrm{a}}\right)\left(c a .100 \mathrm{~kJ} \mathrm{~mol}^{-1}\right)$. In general, the linearly conjugated compounds 1-QC and 4-QC have a lower $E_{\mathrm{a}}$ than the cross-conjugated compounds 2-QC and 3-QC, hence they have shorter half-lives. The NBD-QC photoisomerization quantum yields $\left(\Phi_{\mathrm{NBD} \rightarrow \mathrm{QC}}\right)$ of 1-4 were measured using a Ferrioxalate actinometer ${ }^{41}$ (see ESI, $\dagger$ Section IV). The results are presented in Table 1 . The cross-conjugated compounds 2-NBD and 3-NBD are found to have higher photoisomerization quantum yields in comparison to the corresponding linearly conjugated compounds $1-\mathrm{NBD}$ and 4 -NBD, respectively. In particular, 3-NBD stands out with a photoisomerization quantum yield of around $80 \%$.

\section{Steady state fluorescence spectra, fluorescence quantum yields} and lifetimes

The emission spectra of 1-4 were recorded in toluene and the spectra are shown in Fig. 1. All four compounds show emission upon photoexcitation in the NBD-form. The fluorescence quantum yields of the four compounds in toluene were measured using Coumarin 102 as reference $\left(\Phi_{\mathrm{F}}=0.80\right)$ and the results are shown in Table 1. Out of the four compounds, 4-NBD has the highest fluorescence quantum yield of $49 \%$, followed by 1 -NBD of $4 \%$. This can be compared to the much lower fluorescence quantum yield of $1 \%$ and $0.2 \%$ for the respective cross conjugated compounds 3-NBD and 2-NBD.

To fully understand the underlying cause for such a high variation in the fluorescence quantum yield, the excited state lifetimes of 1-4-NBD were measured using time-correlated single photon counting (TCSPC). The fluorescence decay curves were fitted to a mono- or bi-exponential function, but only the major time constant with the highest amplitude was considered 
(for detailed information see ESI, $\dagger$ Section VI). The result of the measured lifetimes, $\tau_{\mathrm{F}}$, and calculated radiative rate constants, $k_{\mathrm{F}}$, are summarized in Table 1 . Previously, it has been reported that compounds with longer linear conjugation are considered to exhibit stronger fluorescence. ${ }^{42}$ As expected, the longest linearly conjugated compound 4-NBD was found to have the highest fluorescence as well as a longer-lived excited state $(1.28 \mathrm{~ns})$, which is six times longer compared to compound 1-NBD, and more than 50 times longer than the excited state lifetime of compound 2-NBD and 3-NBD. Moreover, since substituted NBDs have higher propensity to undergo photoisomerization to the QC-form on the singlet surface upon direct excitation, ${ }^{43}$ it is worth noting that the photoisomerization and fluorescence quantum yields of compounds 1-4 are complementary, similar to Stilbenes, ${ }^{44}$ i.e. higher $\Phi_{\mathrm{NBD} \rightarrow \mathrm{QC}}$ results in lower $\Phi_{\mathrm{F}}$ and vice versa.

The result of the measured lifetimes, $\tau_{\mathrm{F}}$, and calculated radiative $\left(k_{\mathrm{F}}\right)$ and non-radiative rate constants $\left(k_{\mathrm{nr}}\right)$ are presented in Table 1. The fluorescence rate constants, $k_{\mathrm{F}}=\Phi_{\mathrm{F}} / \tau_{\mathrm{F}}$, of the related NBD-derivatives $\mathbf{1}$ and 2 , and $\mathbf{3}$ and $\mathbf{4}$ are nearly similar. However, the non-radiative rate constants $\left(k_{\mathrm{nr}}\right)$ differ by an order of magnitude between the linearly conjugated compounds 4-NBD and 1-NBD. This difference is about two orders of magnitude between compound 4-NBD and the cross-conjugated compounds 2-NBD and 3-NBD. Time dependent density functional theory (TDDFT) was used to calculate the excited state energies as well as the oscillator strength of transition from ground state to the first singlet excited state for compounds 1-4 in the NBD form, see ESI $\dagger$ for details. The calculated singlet excited state energies are consistent with the trend in the lower energy $\lambda_{\max }$ for the fully conjugated compounds compared to the respective cross-conjugated compounds, as observed in the absorption measurements. Also, the calculated oscillator strength shows that all four compounds have strong and allowed transition from ground state to the first singlet excited state. The oscillator strength is similar for compounds 1-NBD and 2-NBD, and slightly higher for 3-NBD and 4-NBD, which is in line with the radiative rate of fluorescence presented in Table 1 . Generally, the results from emission measurements and TDDFT calculations show that the difference in fluorescence quantum yields of the four compounds are due to the large differences in the nonradiative decay mechanisms (Table 1 ).

Upon photoisomerization to the QC-form, the emission intensity for compounds 1-4 decreases to essentially zero, contrary to those reported by Maafi et al. ${ }^{36,37}$ A plausible explanations for this observed difference is that the emission, in the case of Maafi et al., is due to the aromatic esters, and the fact that NBDs are known quenchers. ${ }^{45}$ Nevertheless, upon photoisomerization, NBDs are progressively converted to QCs, thus diminishing the quenching process. In our case, we did not observe an increase in emission. Hence, the fluorescence can be inferred to arise from the entire conjugated molecule. In addition, during the photoisomerization from NBD to QC, the conjugation becomes interrupted in compounds 1-4 at halfway, since the double bond becomes a single bond. This process turns off the emission in the QC-form, even for the longer conjugated molecules 3-QC and 4-QC. In fact, no emission was measured in the QC-form from any of the four compounds studied.

Once the QC-form is formed, it has several possible mechanistic pathways to revert back to the NBD-form, ${ }^{30-32}$ where one of the most common routes is by thermal activation. ${ }^{27}$ When the QC-form is heated, it relaxes back to the NBD-form. The thermal recovery of fluorescence, going from 4-QC to the corresponding 4-NBD, was recorded at $50{ }^{\circ} \mathrm{C}$ under nitrogen and the result is shown in Fig. 2a. A quantitative recovery of the NBD-form emission was observed at this temperature.

Once modulation of fluorescence was realized upon lightactivated photoisomerization of the NBD-form form to the QC-form, the fluorescence fatigue resistance was tested by carrying out multiple photoisomerization-thermal recovery steps, using 4 as a model compound. The fluorescence measurements for the NBD-form and QC-form were carried out at $6{ }^{\circ} \mathrm{C}$ to slow down
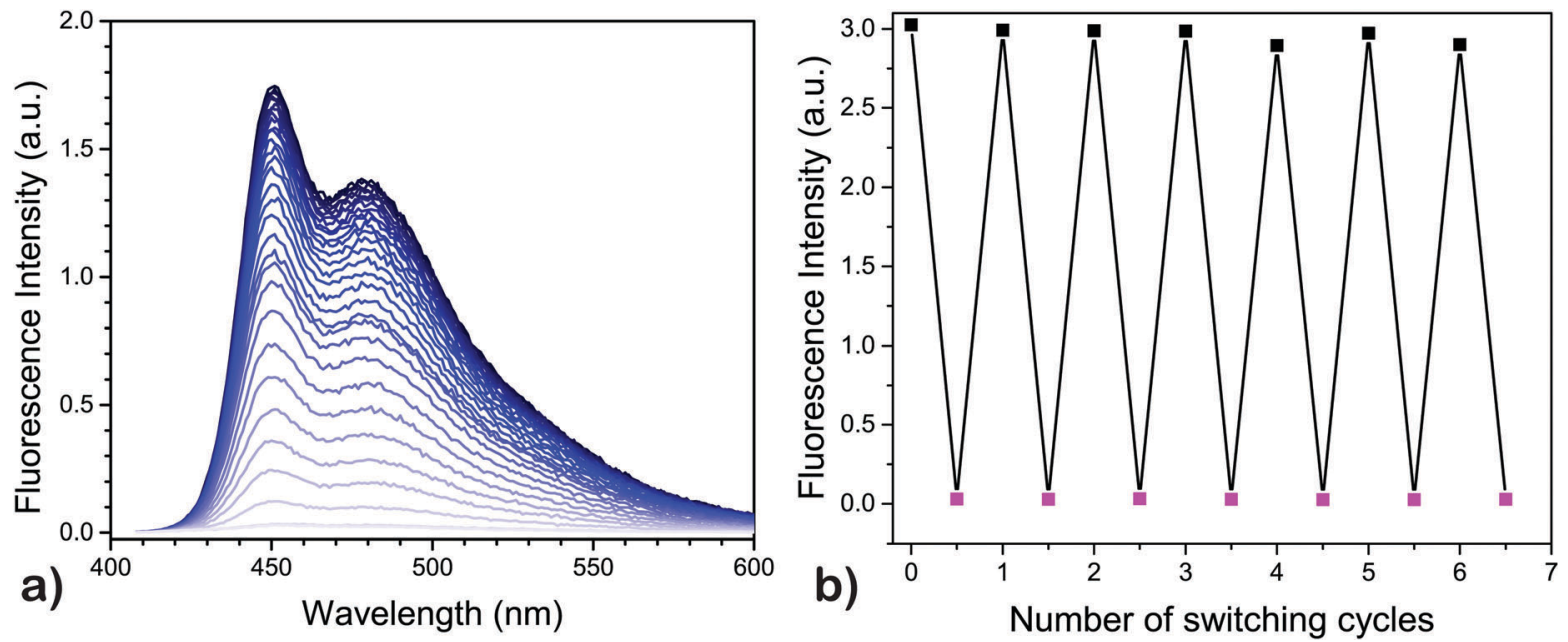

Fig. 2 (a) Fluorescence recovery of $\mathbf{4}$ upon thermal relaxation of the QC-form to NBD-form at $50{ }^{\circ} \mathrm{C}$ in toluene, and (b) cyclability of the modulation of emissive property of 4 in toluene at $6{ }^{\circ} \mathrm{C}$. The fluorescence switching ratio between the NBD-form and QC-form is about 100 . 
the thermal back-conversion of the QC-isomer since the halflife of 4 -QC at $25{ }^{\circ} \mathrm{C}$ is less than $2 \mathrm{~h}$ (Table 1, entry 4). However, at $6{ }^{\circ} \mathrm{C}$, it is well over $8 \mathrm{~h}$, as obtained by extrapolating the data, which was optimal to significantly reduce residual 4-NBD emission for the 4-QC isomer. In between the measurements, the cuvette was kept for 30 minutes at $50{ }^{\circ} \mathrm{C}$ in a heating chamber to thermally recover the NBD-form. About 7 cycles were measured with full recovery of fluorescence of 4-NBD (Fig. 2b). The emission recovery pattern is found to follow a similar trend as the cyclability of photoisomerization of $4 .^{3}$ This means that under nitrogen, compound 4-NBD has virtually no loss of emission. In air, some minor degradation (about $0.2 \%$ per cycle) was observed for compound 4 . $^{3}$

Furthermore, since heat-induced back-isomerization takes a few hours at $25{ }^{\circ} \mathrm{C}$, we investigated light-induced back isomerization of 4-QC to 4-NBD using a $340 \mathrm{~nm}$ LED lamp (Fig. 3). Gratifyingly, the NBD-form was formed readily in less than a minute. This is an important feature that can be used to interconvert the two isomers in short period of time. Although light-induced QC to NBD back-isomerization has been reported previously, ${ }^{32-34}$ our system utilizes a relatively low energy near-UV light. This makes our system attractive for possible biological applications.

In summary, photoisomerization quantum yields of the linearly conjugated 1-NBD and 4-NBD have relatively large values compared to the cross conjugated forms 2-NBD and 3-NBD. In particular, the marked difference between $3-\mathrm{NBD}\left(\Phi_{\mathrm{NBD} \rightarrow \mathrm{QC}}=\right.$ $77 \%)$ and 4 -NBD $\left(\Phi_{\mathrm{NBD} \rightarrow \mathrm{QC}}=15 \%\right)$ is worth noting. Moreover, compounds 1-4 are fluorescent in the NBD-form, with quantum yields ranging from $<1 \%$ to $49 \%$ with 4 -NBD having the highest value. The photoisomerization and fluorescence quantum yields are found to be complementary. Furthermore, the effect of $\pi$-conjugation due to meta/para-arrangement of the bonds in

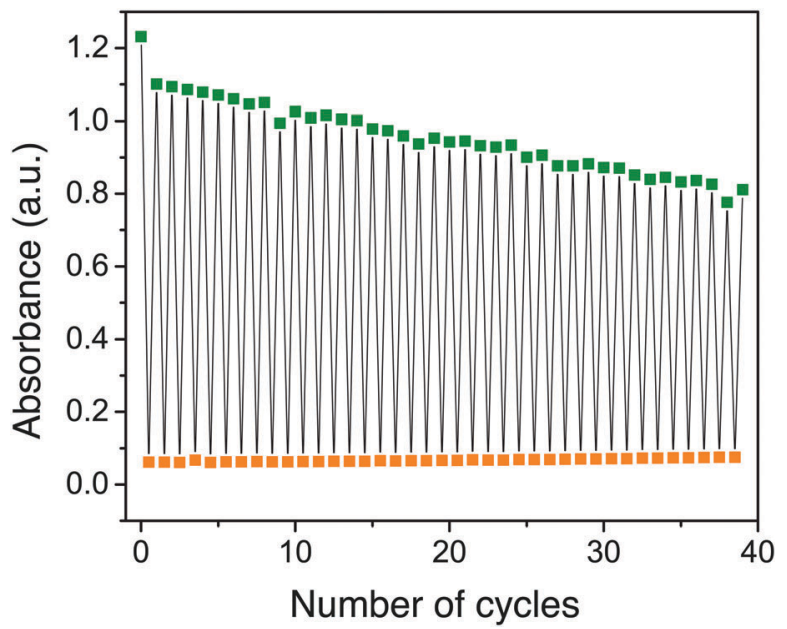

Fig. 3 Dual light switching of compound 4. Upon irradiation with a $405 \mathrm{~nm}$ LED lamp, the NBD form (green) converts to the QC form (orange) in about 60 s. Similarly, the QC form converts to the NBD form readily upon irradiation with a $340 \mathrm{~nm}$ LED lamp while change in absorbance was probed at $430 \mathrm{~nm}$. The rate of degradation per cycle is faster than for the previously reported photo-thermal cycle. ${ }^{3}$ the respective molecules was found to play a significant role, particularly for compounds 3-NBD and 4-NBD.

In contrast, none of the four compounds show any emission from the QC isomer. Emission spectra recorded from the samples after photoisomerization only shows a weak emission profile, which resembles the emission spectrum of the NBDisomer. This emission is therefore assigned to residual NBDform of the molecules that did not photoisomerize. From Fig. 1 it is clear that all four compounds have a wavelength region where the NBD isomer has a high absorptivity. However, the absorptivity of the respective QC isomer is essentially zero at $\lambda_{\text {max } 1}$. This means that all four compounds 1-4 in principle could be photoisomerized $100 \%$ from the NBD to the QC form without any photostationary states. The only limiting factor for complete photoisomerization is the thermally activated backisomerization from QC to NBD. For compound 4-QC, the halflife for the thermal relaxation ranges from about 80 minutes at $25{ }^{\circ} \mathrm{C}$ to less than 6 minutes at $50{ }^{\circ} \mathrm{C}$. Hence, for short-term memory storage applications, compound $\mathbf{4}$ could be a promising photoswitch and its fluorescence emission, which can also be turned off to essentially zero emission by photoisomerization, can be used as a readout. Moreover, we have also demonstrated that it is possible to back-isomerize the QC-form to the NBD-form instantaneously using light, making it an entirely light-activated photoswitch. Thus, the remarkable stability and high fatigue resistance of compound $\mathbf{4}$ demonstrated through a cyclability test (Fig. 2b), particularly under nitrogen, makes it an appealing photochromic system for optical memory storage applications.

\section{Conclusions}

We have synthesized and studied four fatigue resistant NBD/ QC-based negative photochromic systems with conjugated and cross-conjugated electronic arrangements, and investigated their emission properties. The conjugated derivatives are found to be more emissive compared to the cross conjugated analogues. In particular, the linearly conjugated NBD-form of compound 4 is found to show a remarkable fluorescence quantum yield (49\%). This is the highest value for an NBD-based photochromic system that has been reported so far. In contrast, the cross-conjugated analogues show a high photoisomerization quantum yield (about $80 \%)$. We have demonstrated multiple alternating photoactivation and thermal relaxations compound 4 to turn off and recover its emission in the NBD-form. Moreover, in addition to heat, low energy UV (340 nm) light was found to induce the back isomerization of QC-form to the NBD-form in less than a minute, making it a fast-responsive and all-light operated photoswitch system. In particular, compound $\mathbf{4}$, with an all-light activated switching, remarkable fatigue resistance, and high emission, can potentially be used as a vital component in optical memory storages and imaging applications with a fluorescence readout.

\section{Conflicts of interest}

There are no conflicts to declare. 


\section{Acknowledgements}

BET and KMP acknowledge funding from European Research Council (ERC-StG \#37221, SIMONE). NK acknowledges funding from the Swedish Research Council (grant no. 2015-05360). BA and FE acknowledge funding from Swedish Energy Agency.

\section{References}

1 B. Valeur and M. N. Berberan-Santos, J. Chem. Educ., 2011, 88, 731-738.

2 J. R. Lakowicz, Principle of Fluorescence Spectroscopy, Springer, 3rd edn, 2006.

3 B. E. Tebikachew, H. B. Li, A. Pirrotta, K. Borjesson, G. C. Solomon, J. Hihath and K. Moth-Poulsen, J. Phys. Chem. C, 2017, 121, 7094-7100.

4 M. Irie, T. Fukaminato, K. Matsuda and S. Kobatake, Chem. Rev., 2014, 114, 12174-12277.

5 H. Bouas-Laurent and H. Durr, Pure Appl. Chem., 2001, 73, 639-665.

6 T. Tsujioka and M. Irie, J. Photochem. Photobiol., C, 2010, 11, 1-14. 7 R. Klajn, Chem. Soc. Rev., 2014, 43, 148-184.

8 S. Helmy, F. A. Leibfarth, S. Oh, J. E. Poelma, C. J. Hawker and J. R. de Alaniz, J. Am. Chem. Soc., 2014, 136, 8169-8172.

9 I. Yildiz, E. Deniz and F. M. Raymo, Chem. Soc. Rev., 2009, 38, 1859-1867.

10 F. M. Raymo and M. Tomasulo, J. Phys. Chem. A, 2005, 109, 7343-7352.

11 F. M. Raymo and M. Tomasulo, Chem. Soc. Rev., 2004, 34, 327-336.

12 B. Roubinet, M. Weber, H. Shojaei, M. Bates, M. L. Bossi, V. N. Belov, M. Irie and S. W. Hell, J. Am. Chem. Soc., 2017, 139, 6611-6620.

13 J. Fölling, S. Polyakova, V. Belov, A. van Blaaderen, M. L. Bossi and S. W. Hell, Small, 2008, 4, 134-142.

14 J. Cusido, S. S. Ragab, E. R. Thapaliya, S. Swaminathan, J. Garcia-Amoros, M. J. Roberti, B. Araoz, M. M. A. Mazza, S. Yamazaki, A. M. Scott, F. M. Raymo and M. L. Bossi, J. Phys. Chem. C, 2016, 120, 12860-12870.

15 B. Roubinet, M. L. Bossi, P. Alt, M. Leutenegger, H. Shojaei, S. Schnorrenberg, S. Nizamov, M. Irie, V. N. Belov and S. W. Hell, Angew. Chem., Int. Ed. Engl., 2016, 55, 15429-15433.

16 T. Fukaminato, T. Doi, N. Tamaoki, K. Okuno, Y. Ishibashi, H. Miyasaka and M. Irie, J. Am. Chem. Soc., 2011, 133, 4984-4990.

17 E. Murguly, T. B. Norsten and N. R. Branda, Angew. Chem., Int. Ed., 2001, 40, 1752-1755.

18 T. Tsujioka, Mol. Cryst. Liq. Cryst. Sci. Technol., Sect. A, 2006, 344, 51-56.

19 H. Görner, C. Fischer, S. Gierisch and J. Daub, J. Phys. Chem., 1993, 97, 4110-4117.
20 T. Fukaminato, T. Kawai, S. Kobatake and M. Irie, J. Phys. Chem. B, 2003, 107, 8372-8377.

21 H. Qian, B. H. Shao and I. Aprahamian, Tetrahedron, 2017, 73, 4901-4904.

22 Y.-C. Jeong, D. G. Park, I. S. Lee, S. I. Yang and K.-H. Ahn, J. Mater. Chem., 2009, 19, 97-103.

23 Y.-C. Jeong, S. I. Yang, E. Kim and K.-H. Ahn, Tetrahedron, 2006, 62, 5855-5861.

24 T. Kawai, T. Sasaki and M. Irie, Chem. Commun., 2001, 711-712.

25 W. Tan, X. Li, J. Zhang and H. Tian, Dyes Pigm., 2011, 89, 260-265.

26 U. Kakishi, H. Niikura, M. Morimoto, Y. Ishibashi, H. Miyasaka and M. Irie, J. Am. Chem. Soc., 2011, 133, 13558-13564.

27 A. Lennartson, A. Roffey and K. Moth-Poulsen, Tetrahedron Lett., 2015, 56, 1457-1465.

28 M. Quant, A. Lennartson, A. Dreos, M. Kusima, P. Erhart, K. Börjesson and K. Moth-Poulsen, Chem. - Eur. J., 2016, 22, 13265-13274.

29 A. Dreos, K. Börjesson, Z. Wang, A. Roffey, Z. Norwood, D. Kushnir and K. Moth-Poulsen, Energy Environ. Sci., 2016, 10, 728-734.

30 O. Brummel, F. Waidhas, U. Bauer, Y. Wu, S. Bochmann, H.-P. Steinrûk, C. Pap, J. Bachmann and J. Libuda, J. Phys. Chem. Lett., 2017, 8, 2819-2825.

31 S. Miki, T. Maruyama, T. Ohno, T. Tohma, S.-I. Toyama and Z.-I. Yoshida, Chem. Lett., 1988, 861-864.

32 H. Kamogawa and M. Yamada, Macromolecules, 1988, 21, 918-923.

33 T. Nishikubo, A. Kameyama, K. Kishi, T. Kawashima, T. Fujiwara and C. Hijikata, Macromolecules, 1992, 25, 4469-4475.

34 A. Tsubata, T. Uchiyama, A. Kameyama and T. Nishikubo, Macromolecules, 1997, 30, 5649-5654.

35 S. Aiken, R. J. L. Edgar, C. D. Gabbutt, B. M. Heron and P. A. Hobson, Dyes Pigm., 2018, 149, 92-121.

36 M. Maafi, J. J. Aaron and C. Lion, Chem. Lett., 1994, 1865-1868.

37 M. Maafi, C. Lion and J. J. Aaron, Synth. Met., 1996, 83, 167-169.

38 F. Babudri, G. Bilancia, A. Cardone, P. Coppo, L. De Cola, G. M. Farinola, J. W. Hofstraat and F. Naso, Photochem. Photobiol. Sci., 2007, 6, 361-364.

39 K. Stranius and K. Borjesson, Sci. Rep., 2017, 7, 41145.

40 J. N. Demas and G. A. Crosby, J. Phys. Chem., 1971, 75, 991-1024.

41 C. G. Hatchard and C. A. Parker, Proc. R. Soc. London, Ser. A, 1956, 235, 518-536.

42 Y. Yamaguchi, Y. Matsubara, T. Ochi, T. Wakamiya and Z.-I. Yoshida, J. Am. Chem. Soc., 2008, 130, 13867-13869.

43 H. Ikezawa, C. Kutal, K. Yasufuku and H. Yamazaki, J. Am. Chem. Soc., 1986, 108, 1589-1594.

44 S. Malkin and E. Fischer, J. Phys. Chem., 1964, 68, 1153-1163. 45 S. Murov and G. S. Hammond, J. Phys. Chem., 1968, 72, 3797-3801. 